\title{
L'assurance dépendance, une responsabilité essentiellement locale
}

Yuki Sekine

\section{(2) OpenEdition}

1 Journals

\section{Édition électronique}

URL : https://journals.openedition.org/rdctss/2049

DOI : $10.4000 /$ rdctss. 2049

ISSN : 2262-9815

Éditeur

Centre de droit comparé du travail et de la sécurité sociale

\section{Édition imprimée}

Date de publication : 1 avril 2018

Pagination : 144-147

ISSN : 2117-4350

\section{Référence électronique}

Yuki Sekine, "L'assurance dépendance, une responsabilité essentiellement locale », Revue de droit comparé du travail et de la sécurité sociale [En ligne], 1 | 2018, mis en ligne le 01 novembre 2021, consulté le 12 novembre 2021. URL : http://journals.openedition.org/rdctss/2049 ; DOI : https:// doi.org/10.4000/rdctss.2049

\section{(c) (i) (9)}

Revue de droit comparé du travail et de la sécurité sociale est mise à disposition selon les termes de la Licence Creative Commons Attribution - Pas d'Utilisation Commerciale - Pas de Modification 4.0 International. 


\section{YUKI SEKINE}

UNIVERSITÉ DE KOBÉ

\section{L'ASSURANCE DÉPENDANCE, UNE RESPONSABILITÉ ESSENTIELLEMENT LOCALE}

Le 26 mai 2017 a été adoptée la loi ( $n^{\circ} 52$ ) « réformant la loi (de 1997) sur l'assurance dépendance, pour renforcer les programmes locaux intégrés de soins " (Chiiki Houkatsu care-system). Cette réforme s'inscrit dans une continuité visant à consolider les compétences des collectivités locales en matière d'aide aux personnes âgées dépendantes, ainsi qu'à en maîtriser les coûts toujours plus importants. L'on sait que le vieillissement de la population au Japon est très avancé (plus de 30 millions de japonais sont aujourd'hui âgés de plus de 65 ans, soit environ 1 japonais sur 4) et, parmi les défis importants qui en résultent, figure celui de la prise en charge de la dépendance, qui repose au Japon sur un système d'assurance publique mis en place en 2000. Au problème du financement, et donc de la soutenabilité de cette assurance, s'ajoute celui, lié au maintien du niveau de vie et de santé des générations vieillissantes et de leurs familles; c'est à toutes ces questions que les réformes successives, y compris celle de 2017, tentent d'apporter des réponses.

Depuis sa mise en place en 2000, les dépenses liées à l'assurance dépendance n'ont cessé de croître à un rythme qui dépasse les prévisions, atteignant aujourd'hui près du triple de ce qu'elle coûtait au début (de 3600 milliards de yen en 2000, à près de 10000 milliards en 2017), dont résulte une hausse des cotisations à la charge des assurés, lors de chacune des 4 réformes dont elle a fait l'objet. Celles-ci sont versées par l'ensemble de la population âgée de 40 ans et plus, sur des bases différentes pour les personnes âgées de 65 ans et plus (les assurés de la première catégorie), et celles entre 40 et 64 ans (les assurés de la deuxième catégorie). Malgré ces lourdes dépenses, l'aide apportée aux parents dépendants demeure une charge importante pour les familles, notamment pour les aidants familiaux, parfois encore contraints de quitter un emploi stable pour s'occuper d'un parent, et/ou d'un époux.

Les deux lignes directrices de la dernière réforme concernent d'une part l'approfondissement et la promotion des systèmes intégrés de soins locaux (chiiki-houkatsu care system), et d'autre part les mesures financières pour assurer la soutenabilité de l'assurance dépendance. Ces deux principes répondent à leur tour à une préoccupation plus générale qui consiste à promouvoir une culture de cohabitation sociale participative au niveau des municipalités, où chaque habitant prendrait une part plus active à l'action sociale au sein de sa communauté, avec pour objectif, dans ce cas, d'apporter une aide globale et intégrée à l'autonomie des personnes âgées, et lorsqu'elles sont dépendantes, de prévenir l'aggravation de leur état de dépendance.

La première ligne directrice, à savoir l'approfondissement des systèmes intégrés de soins locaux, se décompose en trois types de mesures: 1. Le renforcement des compétences des municipalités (shichou-son) dans la gestion de l'assurance, en élaborant un "plan» de gestion de l'assurance dépendance spécifique, comprenant des mesures destinées spécifiquement à la prévention de l'aggravation de l'état de dépendance, et la promotion de l'autonomie des personnes âgées; 2. La promotion d'une collaboration plus étroite 
entre les soins médicaux et ceux de l'aide à la dépendance, avec la création d'un nouveau type d'établissement destiné à l'hébergement des personnes dépendantes nécessitant des soins médicaux de longue durée, et la collecte de données nécessaires aux départements, pour la réalisation d'une meilleure coordination entre les soins médicaux et ceux relatifs à la dépendance; 3 . La promotion $r$ des initiatives destinées à encourager une culture de cohabitation sociale participative (chiiki-kyousei-shakai) comprenant globalement toutes les politiques de l'action sociale y compris celles destinées aux personnes handicapées, aux enfants, etc., mettant en place des mesures qui permettent aux habitants de collaborer avec les municipalités dans la mise en place d'un système d'aide globale (ou intégrée) englobant tous les aspects de la vie quotidienne, ainsi que la promotion d'établissements combinant les aides aux personnes handicapées de moins de 65 ans, et celles destinées aux personnes âgées dépendantes, permettant par là même une transition plus linéaire entre les deux types d'aides.

Le seconde ligne directrice, consistant à assurer la soutenabilité de l'assurance dépendance, comprend les deux types de mesures suivantes: Augmenter le taux de reste à charge, qui est actuellement de $10 \%$ des coûts en général, et $20 \%$ pour les personnes disposant de revenus élevés (ou plus exactement comparables à la moyenne des personnes actives), pour atteindre $30 \%$ des coûts pour ces derniers; Étendre la base de calcul des cotisations des assurés de la $2^{\text {ème }}$ catégorie (personnes entre 40 et 64 ans) à la rémunération globale, c'est-à-dire, comprenant les primes versées en supplément du salaire, afin d'augmenter encore les recettes, au moins pour les assurés dont les capacités contributives sont jugées suffisantes.

\section{I - LE RENFORCEMENT DES COMPÉTENCES DES MUNICIPALITÉS, GESTIONNAIRES DE L'ASSURANCE DÉPENDANCE}

Afin d'assurer la soutenabilité de l'assurance dépendance à long terme, on considère que les municipalités sont les mieux placées pour analyser les besoins locaux, et mettre en place les mécanismes permettant aux habitants les plus âgés de vivre au sein de leur communauté, en évitant aussi longtemps que possible la perte d'autonomie. Les municipalités sont ainsi invitées, par la nouvelle loi, à analyser les données collectées par les départements, établir des indices d'évaluation, et mettre en place des mesures incitatives envers leurs populations afin de réaliser les objectifs fixés. L'État et les départements effectuent chacun pour leur part des analyses destinées à assister les municipalités dans leurs tâches.

\section{II - CRÉATION D'UN NOUVEAU TYPE D'ÉTABLISSEMENT DESTINÉ À L'HÉBERGEMENT DES PERSONNES DÉPENDANTES NÉCESSITANT DES SOINS MÉDICAUX DE LONGUE DURÉE}

Le coût de l'hospitalisation des personnes âgées connaît une augmentation régulière du fait du vieillissement de la population; c'est pourquoi la maîtrise des dépenses de santé, I'un des défis majeurs liés au vieillissement, repose pour une partie non négligeable sur la gestion de la dépendance, et notamment sur une coordination adéquate entre soins de santé et aide à la dépendance. Une telle coordination, outre l'effet bénéfique qu'elle peut avoir sur l'offre des services, par là mieux adaptée aux besoins des bénéficiaires, permettrait en même temps de réduire les dépenses superflues de santé en les remplaçant par des 
prestations d'aide à la dépendance. C'est dans cette optique que la réforme de 2017, après de longues années de gestation, met en place un nouveau type d'établissement agréé par l'assurance dépendance, destiné à accueillir les personnes âgées dont le niveau de dépendance est élevé et qui nécessitent en même temps des soins hospitaliers de longue durée, le plus souvent du fait d'une maladie chronique. Ce nouveau type d'établissement, dénommé « établissement d'aide clinique et de dépendance »(kaigo-iryou-inn), répondrait à des critères d'habilitation spécifique, à cheval entre un établissement de type hospitalier et de soins à la dépendance. La réforme prévoit qu'à l'issue d'une période transitoire de six ans (fin 2023), les lits actuellement alloués aux personnes âgées dépendantes nécessitant des soins médicaux, dans les établissements hospitaliers seront supprimés et remplacés par les lits alloués aux établissements du nouveau type prévus par la nouvelle loi.

\section{III - PROMOTION DES INITIATIVES POUR L'ENCOURAGEMENT D'UNE CULTURE DE COHABITATION SOCIALE PARTICIPATIVE (CHIIKI-KYOUSEI-SHAKAI) ENGLOBANT L'ENSEMBLE DE L'ACTION SOCIALE}

Ces mesures visent à repenser l'action sociale de manière globale, combinant tous les aspects de la vie quotidienne couverts par l'action sociale, afin d'apporter aux familles qui en ont besoin, une aide compréhensive répondant aux besoins aussi divers que composites pouvant naître au sein de leur collectivité. Pour une meilleure compréhension des besoins spécifiques à leur collectivité et leur population, les municipalités sont invitées à mettre en place des mécanismes de consultation des habitants dans le cadre de l'élaboration de leurs plans d'action sociale, en essayant de mettre en place une aide globale, intégrée, couvrant tous les aspects de la vie quotidienne. À titre d'exemple, la promotion d'établissements combinant les services d'aide aux personnes handicapées de moins de 65 ans, et ceux destinés aux personnes âgées dépendantes, est encouragée, pour permettre une transition plus linéaire et coordonnée entre les deux domaines d'action. Ce type d'établissement pouvant offrir à la fois des prestations d'aide aux personnes handicapées et aux personnes dépendantes, faisant l'objet de deux lois distinctes, feront l'objet de régulations particulières mises en œuvre dès 2018.

\section{IV - RÉVISION DU TAUX DE RESTE À CHARGE POUR LES PERSONNES ÂGÉES DOTÉES DE REVENUS SUFFISANTS}

La première mesure visant à garantir la durabilité et la soutenabilité de l'assurance dépendance, concerne le taux de reste à charge des frais de soins de dépendance. Les aides versées couvrent en principe $90 \%$ des frais liés aux soins de dépendance, alors que les $10 \%$ restants demeurent à la charge de l'assuré. Ce remboursement de $90 \%$ se fait dans la limite des montants plafonds définis par degré de dépendance et par localisation des services (plus coûteux en zone urbaine qu'en zone rurale). Les $10 \%$ à charge de l'assuré sont eux-mêmes plafonnés en fonction des revenus de la personne, mais depuis 2015 (réforme de 2014), ce taux a été porté à 20 \% des frais pour les personnes disposant d'une retraite suffisante, leur permettant d'avoir des ressources suffisantes. Ce taux sera désormais de 30 \% (à partir d'août 2018) pour les assurés les plus aisés, apportant des fonds supplémentaires pour la gestion du système. 


\section{JAPON}

\section{V - LE FINANCEMENT DE L'ASSURANCE DÉPENDANCE}

Afin d'augmenter les recettes de cotisations, le montant de la rémunération servant de base de calcul du montant, pour les assurés « du second alinéa » (ceux entre 40 ans et 64 ans, qui cotisent mais ne bénéficient pas encore des services, à l'exception de ceux âgés entre 60 et 64 ans atteints d'une maladie liée à la vieillesse, selon une liste exhaustive) sera désormais celui des revenus du travail dans leur entièreté, y compris les primes, saisonnières ou de fin d'année, etc. qui seront versées de manière régulière aux salariés. Les contributions de cette catégorie d'assurés assurent $28 \%$ des fonds totaux de l'assurance dépendance, $25 \%$ provenant du budget de l'État, $25 \%$ des collectivités locales, et enfin $22 \%$, des cotisations des assurés de la première catégorie (ceux âgés de 65 ans et plus, pouvant bénéficier de l'aide si leur état de dépendance est reconnu par la commission compétente). Ces cotisations sont prélevées par les assurances maladie, et ensuite versés à l'assurance dépendance. Le fait d'effectuer ce calcul sur une assiette plus large aura pour effet d'augmenter le montant des cotisations afin de mieux maîtriser les finances de l'assurance maladie.

Pour conclure, comme l'on sait qu'il est préférable, pour ne pas aggraver l'état de santé et de dépendance d'une personne âgée en perte d'autonomie, qu'elle puisse continuer à vivre à son domicile, dans un environnement familier, et que les services d'aide soient effectués à domicile (c'est le cas des 3/4 des aides fournies dans le cadre de l'assurance dépendance, seul 1/4 étant des services en établissement), les collectivités locales, et plus spécifiquement les municipalités, ont un rôle important à jouer lorsqu'il s'agit d'identifier les besoins de leur population et d'adapter l'offre de services à ceux-ci. L'objet principal de la réforme de 2017 vise donc à renforcer cette compétence locale, et à impliquer les habitants dans ce processus, en les responsabilisant (par des mesures incitatives), d'une part, mais aussi d'autre part dans le but d'appréhender leurs besoins de manière plus juste, plus précise, et plus englobante. 